IJMMS 26:11 (2001) 671-678

PII. S0161171201010730

http://ijmms.hindawi.com

(c) Hindawi Publishing Corp.

\title{
ON MATRIX TRANSFORMATIONS CONCERNING \\ THE NAKANO VECTOR-VALUED \\ SEQUENCE SPACE
}

\section{SUTHEP SUANTAI}

(Received 15 August 2000 and in revised form 20 October 2000)

\begin{abstract}
We give the matrix characterizations from Nakano vector-valued sequence space $\ell(X, p)$ and $F_{r}(X, p)$ into the sequence spaces $E_{r}, \ell_{\infty}, \underline{\ell}_{\infty}(q), b s$, and $c s$, where $p=\left(p_{k}\right)$ and $q=\left(q_{k}\right)$ are bounded sequences of positive real numbers such that $p_{k}>1$ for all $k \in \mathbb{N}$ and $r \geq 0$.
\end{abstract}

2000 Mathematics Subject Classification. 46A45.

1. Introduction. Let $(X,\|\cdot\|)$ be a Banach space, $r \geq 0$ and $p=\left(p_{k}\right)$ a bounded sequence of positive real numbers. We write $x=\left(x_{k}\right)$ with $x_{k}$ in $X$ for all $k \in \mathbb{N}$. The $X$-valued sequence spaces $c_{0}(X, p), c(X, p), \ell_{\infty}(X, p), \ell(X, p), E_{r}(X, p), F_{r}(X, p)$, and $\underline{\ell}_{\infty}(X, p)$ are defined as

$$
\begin{aligned}
c_{0}(X, p) & =\left\{x=\left(x_{k}\right): \lim _{k \rightarrow \infty}\left\|x_{k}\right\|^{p_{k}}=0\right\}, \\
c(X, p) & =\left\{x=\left(x_{k}\right): \lim _{k \rightarrow \infty}\left\|x_{k}-a\right\|^{p_{k}}=0, \text { for some } a \in X\right\}, \\
\ell_{\infty}(X, p) & =\left\{x=\left(x_{k}\right): \sup _{k}\left\|x_{k}\right\|^{p_{k}}<\infty\right\}, \\
\ell(X, p) & =\left\{x=\left(x_{k}\right): \sum_{k=1}^{\infty}\left\|x_{k}\right\|^{p_{k}}<\infty\right\}, \\
E_{r}(X, p) & =\left\{x=\left(x_{k}\right): \sup _{k} \frac{\left\|x_{k}\right\|^{p_{k}}}{k^{r}}<\infty\right\}, \\
F_{r}(X, p) & =\left\{x=\left(x_{k}\right): \sum_{k=1}^{\infty} k^{r}\left\|x_{k}\right\|^{p_{k}}<\infty\right\}, \\
\underline{\ell}_{\infty}(X, p) & =\bigcap_{n=1}^{\infty}\left\{x=\left(x_{k}\right): \sup _{k}\left\|x_{k}\right\| n^{1 / p_{k}}\right\} .
\end{aligned}
$$

When $X=K$, the scalar field of $X$, the corresponding spaces are written as $c_{0}(p)$, $c(p), \ell_{\infty}(p), \ell(p), E_{r}(p), F_{r}(p)$, and $\underline{\ell}_{\infty}(p)$, respectively. The spaces $c_{0}(p), c(p)$, and $\ell_{\infty}(p)$ are known as the sequence spaces of Maddox. These spaces were first introduced and studied by Simons [7] and Maddox [4,5]. The space $\ell(p)$ was first defined by Nakano [6] and it is known as the Nakano sequence space and the space $\ell(X, p)$ is known as the Nakano vector-valued sequence space. When $p_{k}=1$ for all $k \in \mathbb{N}$, the spaces $E_{r}(p)$ and $F_{r}(p)$ are written as $E_{r}$ and $F_{r}$, respectively. These two 
sequence spaces were first introduced by Cooke [1]. The space $\underline{\ell}_{\infty}(p)$ was first defined by Grosse-Erdmann [2] and he has given in [3] characterizations of infinite matrices mapping between scalar-valued sequence spaces of Maddox. Wu and Liu [10] gave necessary and sufficient conditions for infinite matrices mapping from $c_{0}(X, p)$ and $\ell_{\infty}(X, p)$ into $c_{0}(q)$ and $\ell_{\infty}(q)$. Suantai [8] has given characterizations of infinite matrices mapping $\ell(X, p)$ into $\ell_{\infty}$ and $\underline{\ell}_{\infty}(q)$ when $p_{k} \leq 1$ for all $k \in \mathbb{N}$ and he has also given in [9] characterizations of those infinite matrices mapping from $\ell(X, p)$ into the sequence space $E_{r}$ when $p_{k} \leq 1$ for all $k \in \mathbb{N}$.

In this paper, we extend the results of [8,9] in case $p_{k}>1$ for all $k \in \mathbb{N}$. Moreover, we also give the matrix characterizations from $\ell(X, p)$ and $F_{r}(X, p)$ into the sequence spaces $b s$ and $c s$.

2. Notations and definitions. Let $(X,\|\cdot\|)$ be a Banach space, the space of all sequences in $X$ is denoted by $W(X)$, and $\Phi(X)$ denotes the space of all finite sequences in $X$. When $X=K$, the scalar field of $X$, the corresponding spaces are written as $w$ and $\Phi$.

A sequence space in $X$ is a linear subspace of $W(X)$. Let $E$ be an $X$-valued sequence space. For $x \in E$ and $k \in \mathbb{N}, x_{k}$ stands for the $k$ th term of $x$. For $k \in \mathbb{N}$, we denote by $e_{k}$ the sequence $(0,0, \ldots, 0,1,0, \ldots)$ with 1 in the $k$ th position and by $e$ the sequence $(1,1,1, \ldots)$. For $x \in X$ and $k \in \mathbb{N}$, let $e^{k}(x)$ be the sequence $(0,0, \ldots, 0, x, 0, \ldots)$ with $x$ in the $k$ th position and let $e(x)$ be the sequence $(x, x, x, \ldots)$. We call a sequence space $E$ normal if $\left(t_{k} x_{k}\right) \in E$ for all $x=\left(x_{k}\right) \in E$ and $t_{k} \in K$ with $\left|t_{k}\right|=1$ for all $t_{k} \in \mathbb{N}$. A normed sequence space $(E,\|\cdot\|)$ is said to be norm monotone if $x=\left(x_{k}\right)$, $y=\left(y_{k}\right) \in E$ with $\left\|x_{k}\right\| \leq\left\|y_{k}\right\|$ for all $k \in \mathbb{N}$ we have $\|x\| \leq\|y\|$. For a fixed scalar sequence $\mu=\left(\mu_{k}\right)$, the sequence space $E_{\mu}$ is defined as

$$
E_{\mu}=\left\{x \in W(X):\left(\mu_{k} x_{k}\right) \in E\right\} .
$$

Let $A=\left(f_{k}^{n}\right)$ with $f_{k}^{n}$ in $X^{\prime}$, the topological dual of $X$. Suppose that $E$ is a space of $X$-valued sequences and $F$ a space of scalar-valued sequences. Then $A$ is said to map $E$ into $F$, written by $A: E \rightarrow F$, if for each $x=\left(x_{k}\right) \in E, A_{n}(x)=\sum_{k=1}^{\infty} f_{k}^{n}\left(x_{k}\right)$ converges for each $n \in \mathbb{N}$, and the sequence $A x=\left(A_{n}(x)\right) \in F$. Let $(E, F)$ denote the set of all infinite matrices mapping from $E$ into $F$.

Suppose that the $X$-valued sequence space $E$ is endowed with some linear topology $\tau$. Then $E$ is called a $K$-space if for each $k \in \mathbb{N}$, the $k$ th coordinate mapping $p_{k}: E \rightarrow X$, defined by $p_{k}(x)=x_{k}$, is continuous on $E$. If, in addition, $(E, \tau)$ is a Fréchet (Banach) space, then $E$ is called an FK- (BK-) space. Now, suppose that $E$ contains $\Phi(X)$. Then $E$ is said to have property $\mathrm{AB}$ if the set $\left\{\sum_{k=1}^{n} e^{k}\left(x_{k}\right): n \in \mathbb{N}\right\}$ is bounded in $E$ for every $x=\left(x_{k}\right) \in E$. It is said to have property AK if $\sum_{k=1}^{n} e^{k}\left(x_{k}\right) \rightarrow x$ in $E$ as $n \rightarrow \infty$ for every $x=\left(x_{k}\right) \in E$. It has property $\mathrm{AD}$ if $\Phi(X)$ is dense in $E$.

It is known that the Nakano sequence space $\ell(X, p)$ is an FK-space with property AK under the paranorm $g(x)=\left(\sum_{k=1}^{\infty}\left\|x_{k}\right\|^{p_{k}}\right)^{1 / M}$, where $M=\max \left\{1, \sup _{k} p_{k}\right\}$. If $p_{k}>1$ for all $k \in \mathbb{N}$, then $\ell(X, p)$ is a BK-space with the Luxemburg norm defined by

$$
\left\|\left(x_{k}\right)\right\|=\inf \left\{\varepsilon>0: \sum_{k=1}^{\infty}\left\|\frac{x_{k}}{\varepsilon}\right\|^{p_{k}} \leq 1\right\} .
$$


3. Main results. We first give a characterization of an infinite matrix mapping from $\ell(X, p)$ into $E_{r}$ when $p_{k}>1$ for all $k \in \mathbb{N}$. To do this, we need the following lemma.

LEMMA 3.1. Let $E$ be an $X$-valued BK-space which is normal and norm monotone and let $A=\left(f_{k}^{n}\right)$ be an infinite matrix. Then $A: E \rightarrow E_{r}$ if and only if $\sup _{n} \sum_{k=1}^{\infty}\left|f_{k}^{n}\left(x_{k}\right)\right| / n^{r}$ $<\infty$ for every $x=\left(x_{k}\right) \in E$.

Proof. If the condition holds true, it follows that

$$
\sup _{n} \frac{\left|\sum_{k=1}^{\infty} f_{k}^{n}\left(x_{k}\right)\right|}{n^{r}} \leq \sup _{n} \sum_{k=1}^{\infty} \frac{\left|f_{k}^{n}\left(x_{k}\right)\right|}{n^{r}}<\infty
$$

for every $x=\left(x_{k}\right) \in E$, hence $A: E \rightarrow E_{r}$.

Conversely, assume that $A: E \rightarrow E_{r}$. Since $E$ and $E_{r}$ are BK-spaces, by Zeller's theorem, $A: E \rightarrow E_{r}$ is bounded, so there exists $M>0$ such that

$$
\sup _{\substack{n \in \mathbb{N} \\\left\|\left(x_{k}\right)\right\| \leq 1}} \frac{\left|\sum_{k=1}^{\infty} f_{k}^{n}\left(x_{k}\right)\right|}{n^{r}} \leq M .
$$

Let $x=\left(x_{k}\right) \in E$ be such that $\|x\|=1$. For each $n \in \mathbb{N}$, we can choose a scalar sequence $\left(t_{k}\right)$ with $\left|t_{k}\right|=1$ and $f_{k}^{n}\left(t_{k} x_{k}\right)=\left|f_{k}^{n}\left(x_{k}\right)\right|$ for all $k \in \mathbb{N}$. Since $E$ is normal and norm monotone, we have $\left(t_{k} x_{k}\right) \in E$ and $\left\|\left(t_{k} x_{k}\right)\right\| \leq 1$. It follows from (3.2) that

$$
\sum_{k=1}^{\infty} \frac{\left|f_{k}^{n}\left(x_{k}\right)\right|}{n^{r}}=\frac{\left|\sum_{k=1}^{\infty} f_{k}^{n}\left(t_{k} x_{k}\right)\right|}{n^{r}} \leq M,
$$

which implies

$$
\sup _{n \in \mathbb{N}} \sum_{k=1}^{\infty} \frac{\left|f_{k}^{n}\left(x_{k}\right)\right|}{n^{r}} \leq M .
$$

It follows from (3.4) that for every $x=\left(x_{k}\right) \in E$,

$$
\sup _{n \in \mathbb{N}} \sum_{k=1}^{\infty} \frac{\left|f_{k}^{n}\left(x_{k}\right)\right|}{n^{r}} \leq M\|x\| .
$$

This completes the proof.

THEOREM 3.2. Let $p=\left(p_{k}\right)$ be a bounded sequence of positive real numbers with $p_{k}>1$ for all $k \in \mathbb{N}$ and $1 / p_{k}+1 / q_{k}=1$ for all $k \in \mathbb{N}$, and let $r \geq 0$. For an infinite matrix $A=\left(f_{k}^{n}\right), A \in\left(\ell(X, p), E_{r}\right)$ if and only if there is $m_{0} \in \mathbb{N}$ such that

$$
\sup _{n} \sum_{k=1}^{\infty}\left\|f_{k}^{n}\right\|^{q_{k}} n^{-r q_{k}} m_{0}^{-q_{k}}<\infty
$$

Proof. Let $x=\left(x_{k}\right) \in \ell(X, p)$. By (3.6), there are $m_{0} \in \mathbb{N}$ and $K>1$ such that

$$
\sum_{k=1}^{\infty}\left\|f_{k}^{n}\right\|^{q_{k}} n^{-r q_{k}} m_{0}^{-q_{k}}<K, \quad \forall n \in \mathbb{N} .
$$

Note that for $a, b \geq 0$, we have

$$
a b \leq a^{p_{k}}+b^{q_{k}} .
$$


It follows by (3.7) and (3.8) that for $n \in \mathbb{N}$,

$$
\begin{aligned}
n^{-r}\left|\sum_{k=1}^{\infty} f_{k}^{n}\left(x_{k}\right)\right| & =n^{-r}\left|\sum_{k=1}^{\infty} f_{k}^{n}\left(m_{0}^{-1} \cdot m_{0} x_{k}\right)\right| \\
& \leq \sum_{k=1}^{\infty}\left(n^{-r} m_{0}^{-1}\left\|f_{k}^{n}\right\|\right)\left(\left\|m_{0} x_{k}\right\|\right) \\
& \leq \sum_{k=1}^{\infty} n^{-r q_{k}} m_{0}^{-q_{k}}\left\|f_{k}^{n}\right\|^{q_{k}}+m_{0}^{\alpha} \sum_{k=1}^{\infty}\left\|x_{k}\right\|^{p_{k}} \\
& \leq K+m_{0}^{\alpha} \sum_{k=1}^{\infty}\left\|x_{k}\right\|^{p_{k}}, \quad \text { where } \alpha=\sup _{k} p_{k} .
\end{aligned}
$$

Hence $\sup n^{-r}\left|\sum_{k=1}^{\infty} f_{k}^{n}\left(x_{k}\right)\right|<\infty$, so that $A x \in E_{r}$.

For necessity, assume that $A \in\left(\ell(X, p), E_{r}\right)$. For each $k \in \mathbb{N}$, we have $\sup _{n} n^{-r}\left|f_{k}^{n}(x)\right|$ $<\infty$ for all $x \in X$ since $e^{(k)}(x) \in \ell(X, p)$. It follows by the uniform bounded principle that for each $k \in \mathbb{N}$ there is $C_{k}>1$ such that

$$
\sup _{n} n^{-r}\left\|f_{k}^{n}\right\| \leq C_{k}
$$

Suppose that (3.6) is not true. Then

$$
\sup _{n} \sum_{k=1}^{\infty}\left\|f_{k}^{n}\right\|^{q_{k}} n^{-r q_{k}} m^{-q_{k}}=\infty, \quad \forall m \in \mathbb{N} .
$$

For $n \in \mathbb{N}$, we have by (3.10) that for $k, m \in \mathbb{N}$,

$$
\begin{aligned}
\sum_{j=1}^{\infty}\left\|f_{j}^{n}\right\|^{q_{j}} n^{-r q_{j}} m^{-q_{j}} & =\sum_{j=1}^{k}\left\|f_{j}^{n}\right\|^{q_{j}} n^{-r q_{j}} m^{-q_{j}}+\sum_{j>k}\left\|f_{j}^{n}\right\|^{q_{j}} n^{-r q_{j}} m^{-q_{j}} \\
& \leq \sum_{j=1}^{k} C_{j}^{q_{j}} m^{-q_{j}}+\sum_{j>k}\left\|f_{j}^{n}\right\|^{q_{j}} n^{-r q_{j}} m^{-q_{j}} .
\end{aligned}
$$

This together with (3.11) give

$$
\sup _{n} \sum_{j>k}\left\|f_{j}^{n}\right\|^{q_{j}} n^{-r q_{j}} m^{-q_{j}}=\infty, \quad \forall k, m \in \mathbb{N} .
$$

By (3.13) we can choose $0=k_{0}<k_{1}<k_{2}<\cdots, m_{1}<m_{2}<\cdots, m_{i}>4^{i}$ and a subsequence $\left(n_{i}\right)$ of positive integers such that for all $i \geq 1$,

$$
\sum_{k_{i-1}<j \leq k_{i}}\left\|f_{j}^{n_{i}}\right\|^{q_{j}} n_{i}^{-r q_{j}} m_{i}^{-q_{j}}>2^{i} .
$$

For each $i \in \mathbb{N}$, we can choose $x_{j} \in X$ with $\left\|x_{j}\right\|=1$, for $k_{i-1}<j \leq k_{i}$ such that

$$
\sum_{k_{i-1}<j \leq k_{i}}\left|f_{j}^{n_{i}}\left(x_{j}\right)\right|^{q_{j}} n_{i}^{-r q_{j}} m_{i}^{-q_{j}}>2^{i} .
$$


For each $i \in \mathbb{N}$, let $F_{i}:(0, \infty) \rightarrow(0, \infty)$ be defined by

$$
F_{i}(M)=\sum_{k_{i-1}<j \leq k_{i}}\left|f_{j}^{n_{i}}\left(x_{j}\right)\right|^{q_{j}} n_{i}^{-r q_{j}} M^{-q_{j}}
$$

Then $F_{i}$ is continuous and non-increasing such that $F(M) \rightarrow 0$ as $M \rightarrow \infty$. Thus there exists $M_{i}>0$ such that $M_{i}>m_{i}$ and

$$
F\left(M_{i}\right)=\sum_{k_{i-1}<j \leq k_{i}}\left|f_{j}^{n_{i}}\left(x_{j}\right)\right|^{q_{j}} n_{i}^{-r q_{j}} M_{i}^{-q_{j}}=2^{i} .
$$

Put

$$
y=\left(y_{j}\right), \quad y_{j}=4^{-i} M_{i}^{-\left(q_{j}-1\right)} n_{i}^{-r q_{j} / p_{j}}\left|f_{j}^{n_{i}}\left(x_{j}\right)\right|^{q_{j}-1} x_{j} \text { for } k_{i-1}<j \leq k_{i} .
$$

Thus

$$
\begin{aligned}
\sum_{j=1}^{\infty}\left\|y_{j}\right\|^{p_{j}} & =\sum_{i=1}^{\infty} \sum_{k_{i-1}<j \leq k_{i}} 4^{-i p_{j}} M_{i}^{-p_{j}\left(q_{j}-1\right)} n_{i}^{-r q_{j}}\left|f_{j}^{n_{i}}\left(x_{j}\right)\right|^{p_{j}\left(q_{j}-1\right)} \\
& \leq \sum_{i=1}^{\infty} 4^{-i} \sum_{k_{i-1}<j \leq k_{i}} M_{i}^{-q_{j}} n_{i}^{-r q_{j}}\left|f_{j}^{n_{i}}\left(x_{j}\right)\right|^{q_{j}} \\
& =\sum_{i=1}^{\infty} 4^{-i} \cdot 2^{i} \\
& =\sum_{i=1}^{\infty} \frac{1}{2^{i}}=1 .
\end{aligned}
$$

Thus $y=\left(y_{j}\right) \in \ell(X, p)$. Since $\ell(X, p)$ is a BK-space which is normal and norm monotone under the Luxemburg norm, by Lemma 3.1, we obtain that

$$
\sup _{n} \sum_{k=1}^{\infty} \frac{\left|f_{k}^{n}\left(y_{k}\right)\right|}{n^{r}}<\infty .
$$

But we have

$$
\begin{aligned}
\sup _{n} \sum_{j=1}^{\infty} \frac{\left|f_{j}^{n}\left(y_{j}\right)\right|}{n^{r}} & \geq \sup _{i} \sum_{j=1}^{\infty} \frac{\left|f_{j}^{n_{i}}\left(y_{j}\right)\right|}{n_{i}^{r}} \geq \sup _{i} \sum_{k_{i-1}<j \leq k_{i}} \frac{\left|f_{j}^{n_{i}}\left(y_{j}\right)\right|}{n_{i}^{r}} \\
& =\sup _{i} \sum_{k_{i-1}<j \leq k_{i}} 4^{-i} M_{i}^{-\left(q_{j}-1\right)} n_{i}^{-r\left(q_{j} / p_{j}+1\right)}\left|f_{j}^{n_{i}}\left(x_{j}\right)\right|^{q_{j}} \\
& =\sup _{i} \sum_{k_{i-1}<j \leq k_{i}} 4^{-i} M_{i}^{-\left(q_{j}-1\right)} n_{i}^{-r q_{j}}\left|f_{j}^{n_{i}}\left(x_{j}\right)\right|^{q_{j}} \\
& =\sup _{i} \sum_{k_{i-1}<j \leq k_{i}}\left(\left|f_{j}^{n_{i}}\left(x_{j}\right)\right|^{q_{j}} n_{i}^{-r q_{j}} M_{i}^{-q_{j}}\right) 4^{-i} M_{i} \\
& \geq \sup _{i} 2^{i}=\infty, \text { because } M_{i}>4^{i} .
\end{aligned}
$$

This is contradictory with (3.20). Therefore (3.6) is satisfied. 
THEOREM 3.3. Let $p=\left(p_{k}\right)$ be a bounded sequence of positive real numbers such that $p_{k}>1$ for all $k \in \mathbb{N}, 1 / p_{k}+1 / q_{k}=1$ for all $k \in \mathbb{N}, r \geq 0$ and $s \geq 0$. Then for an infinite matrix $A=\left(f_{k}^{n}\right), A \in\left(F_{r}(X, p), E_{s}\right)$ if and only if there is $m_{0} \in \mathbb{N}$ such that

$$
\sup _{n} \sum_{k=1}^{\infty}\left(k^{-r q_{k} / p_{k}}\left\|f_{k}^{n}\right\|^{q_{k}} n^{-s q_{k}} m_{0}^{-q_{k}}\right)<\infty .
$$

Proof. Since $F_{r}(X, p)=\ell(X, p)_{\left(k^{r / p_{k}}\right)}$, it is easy to see that

$$
A \in\left(F_{r}(X, p), E_{s}\right) \Longleftrightarrow\left(k^{-r / p_{k}} f_{k}^{n}\right)_{n, k} \in\left(\ell(X, p) E_{s}\right) .
$$

By Theorem 3.2, we have $\left(k^{-r / p_{k}} f_{k}^{n}\right)_{n, k} \in\left(\ell(X, p) E_{s}\right)$ if and only if there is $m_{0} \in \mathbb{N}$ such that

$$
\sup _{n} \sum_{k=1}^{\infty}\left(k^{-r q_{k} / p_{k}}\left\|f_{k}^{n}\right\|^{q_{k}} n^{-s q_{k}} m_{0}^{-q_{k}}\right)<\infty .
$$

Thus the theorem is proved.

Since $E_{0}=\ell_{\infty}$, the following two results are obtained directly from Theorems 3.2 and 3.3 , respectively.

COROLLARY 3.4. Let $p=\left(p_{k}\right)$ be a bounded sequence of positive real numbers with $p_{k}>1$ for all $k \in \mathbb{N}$ and let $1 / p_{k}+1 / q_{k}=1$ for all $k \in \mathbb{N}$. Then for an infinite matrix $A=\left(f_{k}^{n}\right), A \in\left(\ell(X, p), \ell_{\infty}\right)$ if and only if there is $m_{0} \in \mathbb{N}$ such that

$$
\sup _{n} \sum_{k=1}^{\infty}\left\|f_{k}^{n}\right\|^{q_{k}} m_{0}^{-q_{k}}<\infty .
$$

COROLLARY 3.5. Let $p=\left(p_{k}\right)$ be a bounded sequence of positive real numbers with $p_{k}>1$ for all $k \in \mathbb{N}$ and let $1 / p_{k}+1 / q_{k}=1$ for all $k \in \mathbb{N}$. Then for an infinite matrix $A=\left(f_{k}^{n}\right), A \in\left(F_{r}(X, p), \ell_{\infty}\right)$ if and only if there is $m_{0} \in \mathbb{N}$ such that

$$
\sup _{n} \sum_{k=1}^{\infty}\left(k^{-r q_{k} / p_{k}}\left\|f_{k}^{n}\right\|^{q_{k}} m_{0}^{-q_{k}}\right)<\infty .
$$

THEOREM 3.6. Let $p=\left(p_{k}\right)$ and $q=\left(q_{k}\right)$ be bounded sequences of positive real numbers with $p_{k}>1$ for all $k \in \mathbb{N}$ and let $1 / p_{k}+1 / t_{k}=1$ for all $k \in \mathbb{N}$. Then for an infinite matrix $A=\left(f_{k}^{n}\right), A \in\left(\ell(X, p), \underline{\ell}_{\infty}(q)\right)$ if and only if for each $r \in \mathbb{N}$, there is $m_{r} \in \mathbb{N}$ such that

$$
\sup _{n, k} \sum_{k=1}^{\infty} r^{t_{k} / a_{n}}\left\|f_{k}^{n}\right\|^{t_{k}} m_{r}^{-t_{k}}<\infty .
$$

PROOF. Since $\underline{\ell}_{\infty}(q)=\cap_{r=1}^{\infty} \ell_{\infty}\left(r^{\left.1 / q_{k}\right)}\right.$, it follows that

$$
A \in\left(\ell(X, p), \underline{\ell}_{\infty}(q)\right) \Longleftrightarrow A \in\left(\ell(X, p), \ell_{\infty}\left(r^{\left.1 / q_{k}\right)}\right), \quad \forall r \in \mathbb{N} .\right.
$$

It is easy to show that for $r \in \mathbb{N}$,

$$
A \in\left(\ell(X, p), \ell_{\infty}\left(r^{\left.1 / q_{k}\right)}\right) \Longleftrightarrow\left(r^{1 / q_{n}} f_{k}^{n}\right)_{n, k} \in\left(\ell(X, p), \ell_{\infty}\right) .\right.
$$


We obtain by Corollary 3.4 that for $r \in \mathbb{N},\left(r^{1 / q_{n}} f_{k}^{n}\right)_{n, k} \in\left(\ell(X, p), \ell_{\infty}\right)$ if and only if there is $m_{r} \in \mathbb{N}$ such that

$$
\sup _{n} \sum_{k=1}^{\infty} r^{t_{k} / q_{n}}\left\|f_{k}^{n}\right\|^{t_{k}} m_{r}^{-t_{k}}<\infty .
$$

Thus the theorem is proved.

THEOREM 3.7. Let $p=\left(p_{k}\right)$ and $q=\left(q_{k}\right)$ be bounded sequences of positive real numbers with $p_{k}>1$ for all $k \in \mathbb{N}$ and let $1 / p_{k}+1 / t_{k}=1$ for all $k \in \mathbb{N}$. For an infinite matrix $A=\left(f_{k}^{n}\right), A \in\left(F_{r}(X, p), \underline{\ell}_{\infty}(q)\right)$ if and only if for each $i \in \mathbb{N}$, there is $m_{i} \in \mathbb{N}$ such that

$$
\sup _{n} \sum_{k=1}^{\infty} i^{t_{k} / q_{n}} k^{-r t_{k} / p_{k}}\left\|f_{k}^{n}\right\|^{t_{k}} m_{i}^{-t_{k}}<\infty .
$$

Proof. Since $F_{r}(X, p)=\ell(X, p)_{\left(k^{r / p_{k}}\right)}$, it implies that

$$
A \in\left(F_{r}(X, p), \underline{\ell}_{\infty}(q)\right) \Longleftrightarrow\left(k^{-r / p_{k}} f_{k}^{n}\right)_{n, k} \in\left(\ell(X, p), \underline{\ell}_{\infty}(q)\right) .
$$

It follows from Theorem 3.6 that $A \in\left(F_{r}(X, p), \underline{\ell}_{\infty}(q)\right)$ if and only if for each $i \in \mathbb{N}$, there is $m_{i} \in \mathbb{N}$ such that

$$
\sup _{n} \sum_{k=1}^{\infty} i^{t_{k} / q_{n}} k^{-r t_{k} / p_{k}}\left\|f_{k}^{n}\right\|^{t_{k}} m_{i}^{-t_{k}}<\infty .
$$

THEOREM 3.8. Let $p=\left(p_{k}\right)$ be bounded sequence of positive real numbers with $p_{k}>1$ for all $n \in \mathbb{N}$ and let $1 / p_{k}+1 / q_{k}=1$ for all $k \in \mathbb{N}$. Then for an infinite matrix $A=\left(f_{k}^{n}\right), A \in(\ell(X, p), b s)$ if and only if there is $m_{0} \in \mathbb{N}$ such that

$$
\sup _{n} \sum_{k=1}^{\infty}\left\|\sum_{i=1}^{n} f_{k}^{i}\right\|^{q_{k}} m_{0}^{-q_{k}}<\infty .
$$

Proof. For an infinite matrix $A=\left(f_{k}^{n}\right)$, we can easily show that

$$
A \in(\ell(X, p), b s) \Longleftrightarrow\left(\sum_{i=1}^{n} f_{k}^{i}\right)_{n, k} \in\left(\ell(X, p), \ell_{\infty}\right) .
$$

This implies by Corollary 3.4 that $A \in(\ell(X, p), b s)$ if and only if there is $m_{0} \in \mathbb{N}$ such that

$$
\sup _{n} \sum_{k=1}^{\infty}\left\|\sum_{i=1}^{n} f_{k}^{i}\right\|^{q_{k}} m_{0}^{-q_{k}}<\infty .
$$

THEOREM 3.9. Let $p=\left(p_{k}\right)$ be a bounded sequence of positive real numbers with $p_{k}>1$ for all $k \in \mathbb{N}$ and let $1 / p_{k}+1 / q_{k}=1$ for all $k \in \mathbb{N}$. Then for an infinite matrix $A=\left(f_{k}^{n}\right), A \in(\ell(X, p), c s)$ if and only if

(1) there is $m_{0} \in \mathbb{N}$ such that $\sup _{n} \sum_{k=1}^{\infty}\left\|\sum_{i=1}^{n} f_{k}^{i}\right\| q_{k} m_{0}^{-q_{k}}<\infty$ and

(2) for each $k \in \mathbb{N}$ and $x \in X, \sum_{n=1}^{\infty} f_{k}^{n}(x)$ converges. 
Proof. The necessity is obtained by Theorem 3.8 and by the fact that $e^{(k)}(x) \in$ $\ell(X, p)$ for every $k \in \mathbb{N}$ and $x \in X$.

Now, suppose that (1) and (2) hold. By Theorem 3.8, we have $A: \ell(X, p) \rightarrow b s$. Let $x=$ $\left(x_{k}\right) \in \ell(X, p)$. Since $\ell(X, p)$ has the AK property, we have $x=\lim _{n \rightarrow \infty} \sum_{k=1}^{n} e^{(k)}\left(x_{k}\right)$. By Zeller's theorem, $A: \ell(X, p) \rightarrow b s$ is continuous. It implies that

$$
A x=\lim _{n \rightarrow \infty} \sum_{k=1}^{n} A e^{(k)}\left(x_{k}\right) .
$$

By (2), $A e^{(k)}\left(x_{k}\right) \in c s$ for all $k \in \mathbb{N}$. Since $c s$ is a closed subspace of $b s$, it implies that $A x \in c s$, that is, $A: \ell(X, p) \rightarrow c s$.

ACKNOWLEDGEMENT. The author would like to thank the Thailand Research Fund for the financial support during the preparation of this paper.

\section{REFERENCES}

[1] R. G. Cooke, Infinite Matrices and Sequence Spaces, Macmillan, London, 1950. MR 12,694d. Zbl 040.02501.

[2] K.-G. Grosse-Erdmann, The structure of the sequence spaces of Maddox, Canad. J. Math. 44 (1992), no. 2, 298-307. MR 93d:46017. Zbl 777.46008.

[3] _ Matrix transformations between the sequence spaces of Maddox, J. Math. Anal. Appl. 180 (1993), no. 1, 223-238. MR 95d:40017. Zbl 791.47029.

[4] I. J. Maddox, Spaces of strongly summable sequences, Quart. J. Math. Oxford Ser. (2) 18 (1967), 345-355. MR 36\#4195. Zbl 156.06602.

[5]___ Paranormed sequence spaces generated by infinite matrices, Proc. Cambridge Philos. Soc. 64 (1968), 335-340. MR 36\#5565. Zbl 157.43503.

[6] H. Nakano, Modulared sequence spaces, Proc. Japan Acad. 27 (1951), 508-512. MR 13,954g. Zbl 044.11302.

[7] S. Simons, The sequence spaces $l\left(p_{v}\right)$ and $m\left(p_{v}\right)$, Proc. London Math. Soc. (3) 15 (1965), 422-436. MR 31\#600. Zbl 128.33805.

[8] S. Suantai, On matrix transformations related to Nakano vector-valued sequence spaces, Bull. Calcutta Math. Soc. 91 (1999), no. 3, 221-226. MR 2000m:46041. Zbl 943.47025.

[9] S. Suantai and C. Sudsukh, Matrix transformations of Nakano vector-valued sequence space, Kyungpook Math. J. 40 (2000), no. 1, 93-97. MR 2001g:46041. Zbl 992.06378.

[10] C. X. Wu and L. Liu, Matrix transformations on some vector-valued sequence spaces, Southeast Asian Bull. Math. 17 (1993), no. 1, 83-96. MR 94e:46041. Zbl 794.40006.

Suthep Suantai: Department of Mathematics, Faculty of Science, Chaing Mai UniVERSITY, CHIANG MAI, 50200, THAILAND

E-mail address: ma1suthe@science. cmu.ac.th 


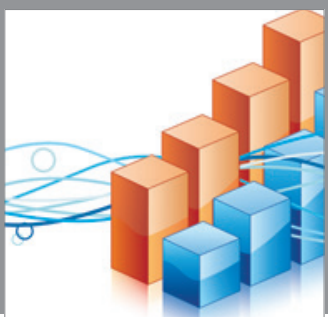

Advances in

Operations Research

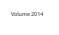

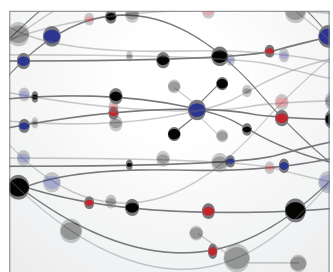

\section{The Scientific} World Journal
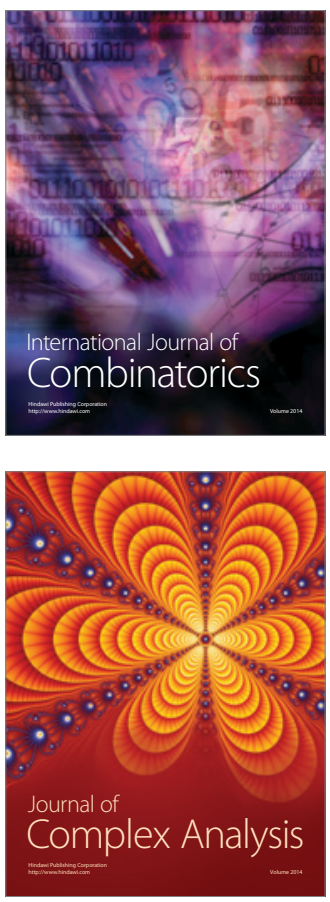

International Journal of

Mathematics and

Mathematical

Sciences
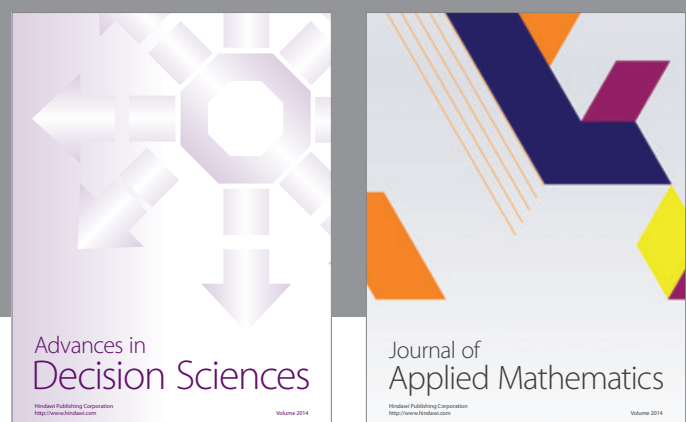

Journal of

Applied Mathematics
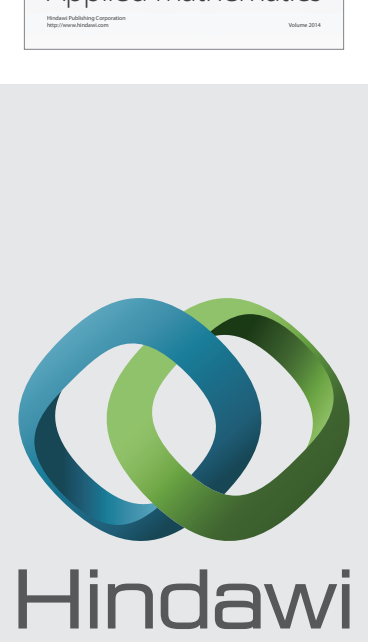

Submit your manuscripts at http://www.hindawi.com
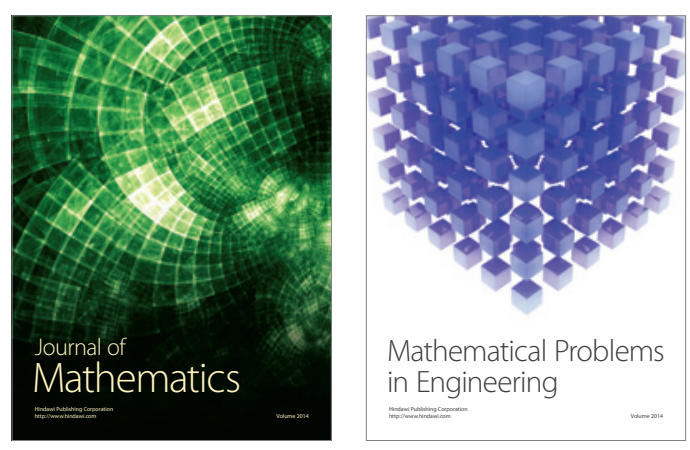

Mathematical Problems in Engineering
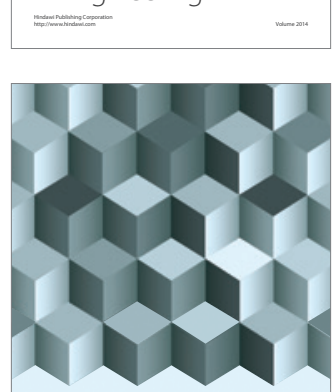

Journal of

Function Spaces
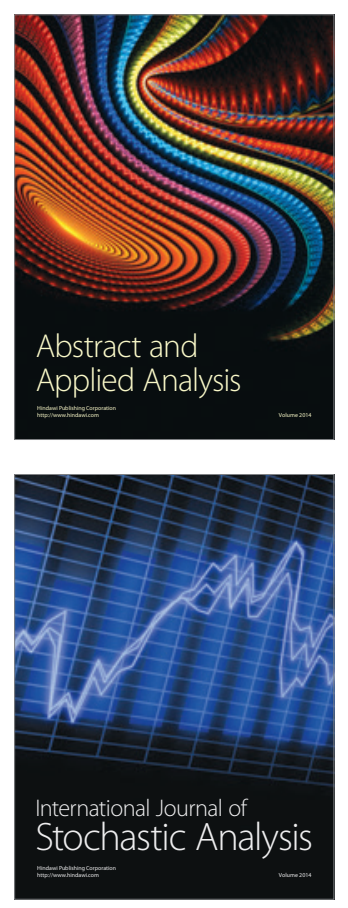

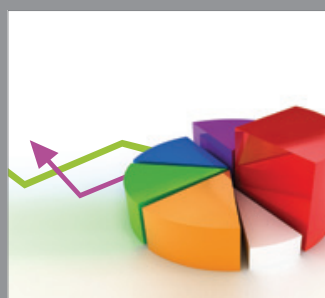

ournal of

Probability and Statistics

Promensencen
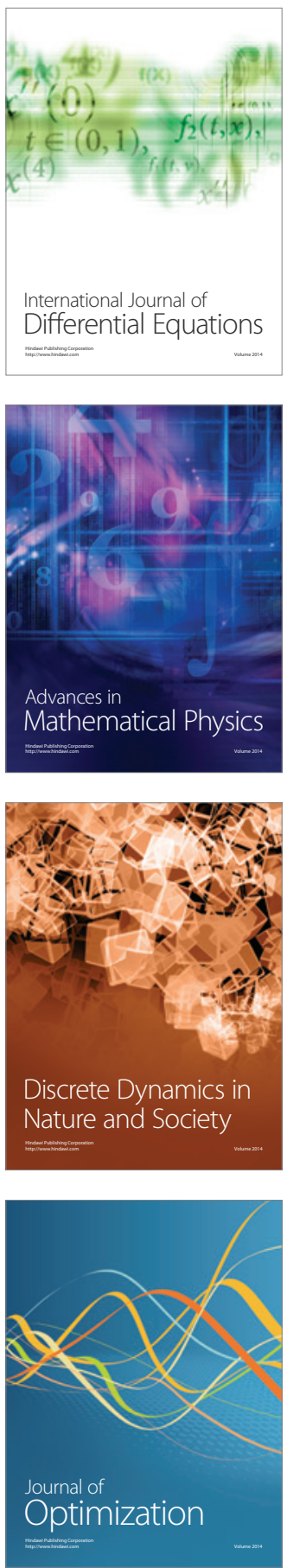